\title{
INVESTIGATION OF MINORITY CARRIER RECOMBINATION IN GaAs: Sn BY MEANS OF EBIC AND CL
}

G. OELGART and N. PUHLMANN*

Sektion Physik der Universität Leipzig, Linnèstrasse 5, D-7010 Leipzig, Germany

*Sektion Physik der Humboldt-Universität zu Berlin, Invalidenstrasse 110, D-1040 Berlin, Germany

\section{Abstract}

The recombination properties of minority carriers have been determined from EBIC and CL measurements versus the primary electron beam energy on step cooling LPE grown n-type GaAs:Sn $\left(3 * 10^{16} \mathrm{~cm}^{-3} \leq \mathrm{N}_{D}-\mathrm{N}_{\mathrm{A}} \leq 7 * 10^{18} \mathrm{~cm}^{-3}\right.$ ) in the temperature range $85 \mathrm{~K} \leq \mathrm{T} \leq$ $300 \mathrm{~K}$. Based on the 1 ifetime and internal quantum yield analysis as a function of the electron concentration we determined the radiative recombination coefficient to $B^{*} 5 * 10^{-11} \mathrm{~cm}^{3} \mathrm{~s}^{-1}$ and the internal quantum yield increases from $3 \%\left(n_{0}=3 * 10^{16} \mathrm{~cm}^{-3}\right)$ to $30 \%$ $\left(n_{0}=8 * 10^{17} \mathrm{~cm}^{-3}\right)$ at room temperature. The increase in external luminescence strength versus diminished temperature is caused by the lowered self-absorption and by the rising internal quantum efficiency. Based on the diffusion length and internal quantum $y i e l d$ analysis vs the temperature, the non-radiative recombination due to the deep Sn-acceptor was shown on strongly doped samples $\left(\mathrm{n} \geq 10^{18} \mathrm{~cm}^{-3}\right.$ ) in the range $\mathrm{T} \leq 200 \mathrm{~K}$.

\section{Theoretical Fundamentals}

The record of EBIC strength versus the primary electron beam energy $\left(\mathrm{I}_{\mathrm{EBIC}}\left(\mathrm{E}_{\mathrm{pr}}\right)\right.$ ) on a Schottky barrier (cf Fig. $1 \mathrm{~b}$ ) is a well established tool for the determination of minority carrier diffusion length's (L) $h$ comparable with the range of the depth dose function The total current can be expressed by:

$$
I_{E B I C}=I_{S C R}+I_{n}
$$

$I_{S C R}$ and $I_{n}$ are EBIC contributions due to excess carriers generated in the space charge region and the neutral n-region. respectively, which are given in $/ 1 /$. Normally, the charge 
collection efficiency $(n)$, defined as the EBIC strength normalized to the total number of generated electron-hole pairs $G_{0}$, is used:

$$
\eta=I_{E B I C} / G_{0} \quad G_{0}=s_{0}^{\infty} \phi(z) d z
$$

where $\phi(z)$ denotes the depth distribution function of electron hole pair generation.



In the following we consider the CL signal from a semi-infinite semiconductor covered by a dead layer of the thickness $z_{t}$ (cf Fig. 1a). The CL intensity ( $I_{C L}$ ) can be calculated using the relation /2/:

Fig. 1

$$
\begin{aligned}
& I_{C L}=2 \pi G_{0} \frac{\tau}{\tau} h{ }_{g}^{\theta} c_{d \theta}^{c} \sin \theta \frac{\exp \left(-\alpha z_{t}\right)}{1-\left(\alpha L_{h}\right)^{2}}{ }_{z_{t}}^{\infty} d z \exp \left[-\alpha\left(z-z_{t}\right)\right] \phi(z)- \\
& -\frac{a L_{h}+S}{1+S}{ }_{z_{t}}^{\infty} d z \exp \left[\left(z_{t}-z\right) / L_{h}\right] \phi(z)
\end{aligned}
$$

Where a denotes the selfabsorption coefficient divided by $\cos \theta_{C}$. $\theta_{c}$ the critical angle of total reflection at the surface: $\tau_{h} \cdot{ }^{\tau_{r}}{ }^{-}$ the total and radiative carrier lifetime, respectively, and $S=$ $v_{s} / v_{d}$ - the surface recombination velocity $\left(v_{s}\right)$ normalized to the diffusion velocity $v_{d}=L / \tau$. For the depth distribution $\phi(z)$ we use the expression given by /3,4/:

$$
\phi(z)=\exp -\left[(z-a) / z_{0}\right]^{2}+\exp -\left(z / z_{1}\right)^{2}
$$

with

$(\mathrm{a} / \mu \mathrm{m})=0.00267\left(\mathrm{E}_{\mathrm{pr}} / \mathrm{keV}\right)^{1.635} ; \mathrm{z}_{0}=2.416 \mathrm{a} ; \mathrm{z}_{1}=0.472 \mathrm{a}$

The relation (3) describes a monochromatic CL signal for the wave length's $\lambda$ within the spectral range of the photon distribution $f(h \nu)$ generated in the semiconductor. Indeed, in the experiments 
to discuss here the spectral unresolved CL signal was recorded with nearly energy independent detector sensitivity in the spectral range $f(h \nu)$. Therefore, the determined absorption coefficients are "effective" one.

Results and Discussion

Fig. 2 displays the normalized collection efficiency $\eta=\eta\left(E_{p r}\right) / \eta_{0}$

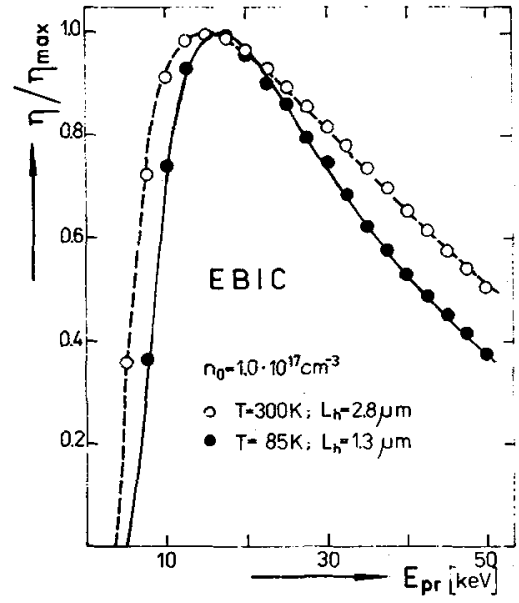

$\bar{F}$ ig. 2

$\left(n_{0^{-}}\right.$maximum value of $\eta\left(E_{p r}\right)$ versus the primary energy of the electron beam ( $E_{p r}$ ) observed at room temperature (open circles) and at $T=85 \mathrm{~K}$ (closed circles). The theoretical computations based on the model given in /1/ excellently align the experimental data points providing the diffusion length given in the Fig. 2 .

Fig. 3 shows the dependencies $I_{C L}\left(E_{p r}\right)$ (normalized to the total yeneration rate) experimentally shserved at $\mathrm{T}=300 \mathrm{~K}$ (open

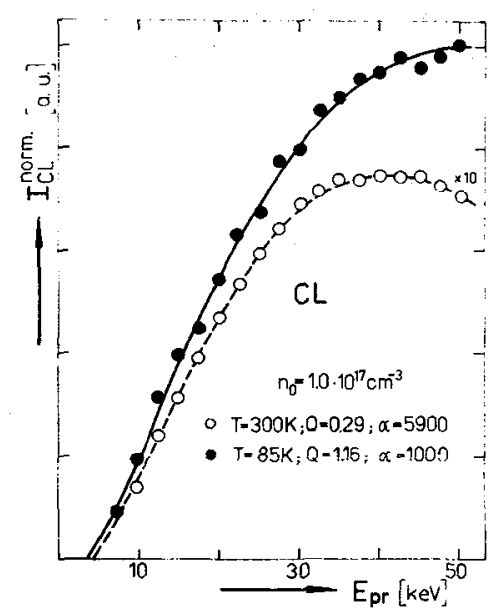
circles) and at $\mathrm{T}=85 \mathrm{~K}$ (closed circles) as well as the theoretical dependencies calculated using equation (3). starting from $L_{h}$ the theoretical data were fitted to the experimentally observed results by the parameters $Q$ and $\alpha$. representing the relative internal quantum efficiency of one sample with regard to the others and the averaged self-absorption coefficient (cf. Fig. 3).

We relate the diffusion length

Fig. 3 
and the total lifetime $\tau_{h}$ using the relations $L_{h}=\left(D_{h}{ }^{*} \tau_{h}\right)^{1 / 2}$ and $D=\mu k T / e\left(\mu-\right.$ mobility). As a result the values ${ }^{\tau_{h}}$ and $Q$ are 1 isted in Fig. 4 versus $N_{D}-N_{A}$. Three $\tau_{h}$-ranges can be distinguished with regard to the effective donor concentration:

(i) $r_{h}$ is nearly independent of $N_{D}-N_{A}$ for $n_{0}<3.5 * 10^{17} \mathrm{~cm}^{-3}$. indicating that the decay of excess holes in the various samples is dominated by an identical nonradiative recombination mechanism. which we attribute to the Shockley-Read-Hall recombination.

(ii) For $n_{0} \geq 10^{18} \mathrm{~cm}^{-3}$ the total lifetime can be approximated by the relation $\tau_{A}=1 /\left(n_{0}^{2} * C_{A}\right)$ typical for an Auger-process.

(iii) In the intermediate cange $\left(3 ! 5 * 10^{17} \mathrm{~cm}^{-3} \leq \mathrm{n}_{0}<1 * 10^{18} \mathrm{~cm}^{-3}\right) \tau_{h}$ decreases and $Q$ simultaneously increases. This behavior is caused by the radiative lifetime $\left(\tau_{r}=1 /\left(n_{0}{ }^{*} B\right)\right)$ comparable with the nonradiative one.

The total lifetime composed by the three parts mentioned above $\left(1 / \tau_{h}=1 / \tau_{\mathrm{SRH}}+1 / \tau_{r}+1 / \tau_{A}\right)$ obeys the relation in the low-injection limit:

$$
\tau_{h}=1 /\left(c_{p} * v_{t h} * N_{t}+B * n_{0}+C_{a} * n_{0}^{2}\right)
$$

Where $c_{p}$ and $N_{t}$ denotes the capture cross section and the concentration of the hole lraps, respectively. $v_{t h}$ is the thermic velooity of excess carriers and $B$ and $C_{A}$ are the radiative and Auger recombination constant, respectively.

In a least square fit procedure the equations (5) and the internal quantum yield $n_{i}=\tau_{h} / \tau$ were fitted to the experimentally observed dependencies $\tau_{h}\left(n_{0}\right)$ and $Q\left(n_{0}\right)$, respectively (Fig's 4 and 5 ).

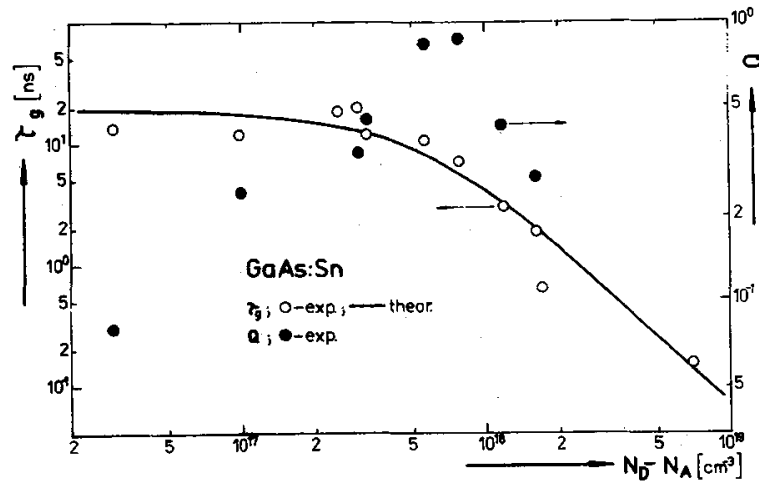

Fig. 4

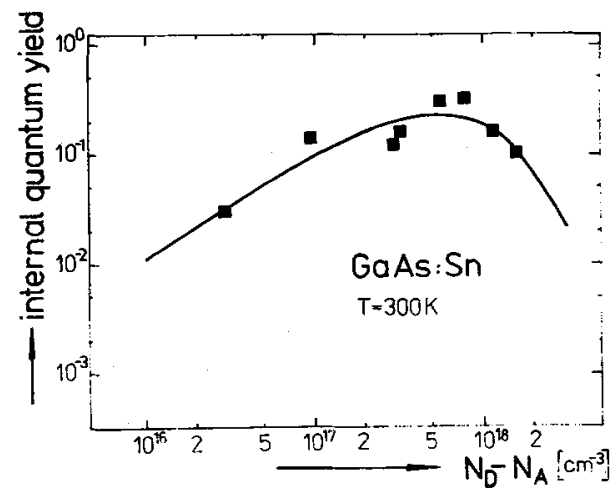

Fig. 5

The close agreement between the experimental and calculated 
concentration dependencies of $\tau h_{15}\left(n_{0}\right)$ and $Q\left(n_{0}\right)$ permits evaluation of the three parameters $N_{t}=5 * 10^{15} \mathrm{~cm}^{-3}, B=5 * 10^{-11} \mathrm{~cm}^{3} \mathrm{~s}^{-1}$, and $\mathrm{C}_{\mathrm{A}}=$ $1.5 \times 10^{-28} \mathrm{~cm}^{6} \mathrm{~s}^{-1}$. The trap concentration $\mathrm{N}_{t}$ follows in conjunction with the capture cross-section $c_{p}=1 * 10^{-15} \mathrm{~cm}^{2}$.

At low temperatures the high energy tail of $n / n_{\max }$ stronger drops with regard to the room temperature results representing the diminished diffusion length (cf. Fig. 2). Whereas the self-absorption coefficient decreases an increase of the relative internal quantum yield was observed with diminished temperature (cf. Fig. 3). The shorter diffusion length and smaller a value produce a decrease of the self-absorption and in conjunction with the increasing quantum yield a strong increase (more than a factor 10) of the external CL-intensity can be observed at lowered temperature (cf. Fig. 3).

The amphoteric behavior of the $S n$ impurity has been well established. In that what follows we will discuss the recombination activity of the tin-acceptor. The hole diffusion lengths on differently doped samples $\left(x-n_{0}=3 * 10^{16} \mathrm{~cm}^{-3} ;\right.$ $1 * 10^{17} \mathrm{~cm}^{-3} ; \Delta-3.3 * 10^{17} \mathrm{~cm}^{-3} ;=-5.7 * 10^{17} \mathrm{~cm}^{-3} ; 0-1.2 * 10^{18} \mathrm{~cm}^{-3}$ ) are listed in Fig. 6 versus temperature. For $N_{D}-N_{A} \leq 10^{17} \mathrm{~cm}^{-3} L_{h}$ exhibits the same variation vs. $T$. With increasing tin incorporation the hole diffusion length decreases to a greater extent with lowered temperature. With the intention to understand these features we compare the diffusion length and internal quantum yield of the samples doped with $1 * 10^{17} \mathrm{~cm}^{-3}$ (open circles) and $1.2 * 10^{18} \mathrm{~cm}^{-3}$ (closed circles) in Fig. 7 .
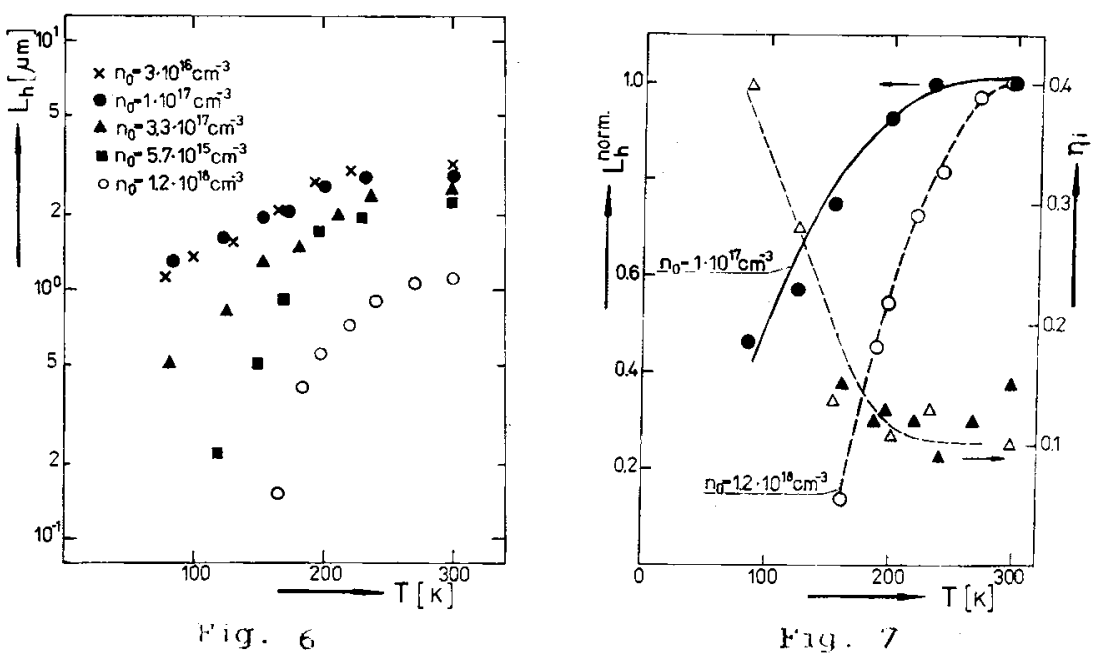
The internal quantum yield of the low doped specimen is nearly constant betwecn $200 \mathrm{~K} \leq \mathrm{T} \leq 300 \mathrm{~K}$ and increases by about a factor four in the range $85 \mathrm{~K} \leq \mathrm{T} \leq 200 \mathrm{~K}$. This fact corresponds to the diffusion length variation vs. the temperature. A simple explanation of these features can be given by the assumption of a decreasing radiative lifetime at temperatures below $200 \mathrm{~K}$. The low temperature quantum yield of 0.4 confirms a value of the radiative lifetime comparable to the nonradiative one. On the higher doped sample $\eta_{i} \approx 0.12$ remains nearly unaltered in the temperature range investigated here. In contrast the $\mathrm{L}_{\mathrm{h}}$-value of the higher doped sample stronger decreases than that of the low doped specimen. This behavior supports the simultaneous orop of both radiative and nonradiative lifetime in the higher doped sample. The shortening of the nonradiative lifetime we tentatively explain by a recombination channel involving the deep tin-acceptor with increasing activity when the temperature decreases.

/1/ Kittler M and Schröder K-W phys.stat.sol.(a) $77 \quad 139$ (1983)

12 / Hergert W, Reck P. Pascmann L, and Schreiber J

$\begin{array}{lllll} & \text { plyss.stat.sol.(a) } & 101 & 611 & \text { (1987) }\end{array}$

/3/ Oelgart G. and Scholz H phys.stat.sol.(a) 75547 (1983)

14/ Koch F. Dissertation. 1988, Humboldt-Universität Berlin 